\title{
Influencia de la Temperatura en la Floración del Algarrobo (Prosopis pallida) en los valles: Bajo Piura, Chira. Región Piura-Perú
}

\section{Influence of the Temperature in the Flowering of the Algarrobo tree (Prosopis pallida) in the valleys: Under Piura, Chira. Piura Región-Perú}

\author{
Ninell Dedios Mimbela \\ Servicio Nacional de Meteorología e Hidrología, Piura, Perú
}

Recibido el 15 de junio del 2017. Revisado el 13 de julio del 2017. Aceptado el 20 de julio del 2017

DOI: https://doi.org/10.33017/RevECIPeru2017.0006/

\section{Resumen}

El presente estudio hace referencia al proceso metodológico aplicado incluido los resultados alcanzados y recomendaciones sobre como la temperatura y su variabilidad influyen en el proceso de floración del algarrobo. La recopilación de estudios realizados sobre el algarrobo (Prosopis pallida) son importantes y necesarias para entender el escenario biofísico en el cual se desarrolla y su proceso de adaptabilidad ante la variabilidad del clima. El objetivo general del estudio se enfoca en evaluar la influencia de la temperatura en la fase de floración de la especie Prosopis pallida (algarrobo) en los valles: Bajo Piura, Chira. Región Piura-Perú. Se encontró una correlación positiva entre el adelanto de la floración con la abundancia de flores en relación al comportamiento de la temperatura y cómo influyen los sectores Niño 1+2 o Niño 3.4 sobre la variabilidad de la temperatura y a su vez sobre el adelanto y/o abundancia de esta fase. Hemos observado en el presente estudio que la floración no es estable tanto estacional como anualmente sino que varía de un año a otro tal y como indican algunos estudios realizados por FAO y algunos autores.

Descriptores: Algarrobo, floración, temperatura

\section{Abstract}

This study refers to the methodology process applied included the results achieved and recommendations as temperature and its variability influence the flowering process of algarrobo. The collection of studies on algarrobo (Prosopis pallida) are important and necessary to understand the biophysical scenario in which the process develops and adaptability to climate variability. The overall objective of the study focuses on assessing the influence of temperature on the flowering phase of Prosopis pallida (algarrobo) in Mallares, Bernal and La Esperanza studies sector. We found a positive correlation between the advancement of the bloom with abundant flowers in relation to the behavior of the temperature and how they influence the sectors Niño $1+2$ and Niño 3.4 on the variability of the temperature and turn on the advancement and / or abundance of this phase. In the present study we observed that flowering is not stable both seasonally and annually but varies from year to year as studies indicated by FAO and some authors.

Keywords: Algarrobo, flowering, temperatura 


\section{Introducción}

En la Región de Piura-Perú el algarrobo Prosopis pallida, es un género característico del bosque seco ecuatorial o bosque estacionalmente seco (BES). Debido a las condiciones ecológicas extremas (xericidad y salinidad), esta especie se encuentra en constante amenaza, siendo vulnerable y corriendo el riesgo de desaparecer por causas antrópicas [1], incluido sus usos tradicionales que también se están perdiendo [2]. El estudio tiene por objetivo analizar la influencia de la temperatura sobre la floración del algarrobo Prosopis pallida, destacando los estudios realizados por Silva \& Higaona [3] en las Pampas de Olmos (Lambayeque), o los realizados por Borchert [4] quienes desarrollaron un amplio estudio sobre la fenología y la periodicidad en la floración de especies neotropicales del bosque seco ecuatorial [5]. Se dice que temperaturas inferiores a $5^{\circ} \mathrm{C}$ originarían la paralización de la circulación de la savia debido a la congelación y por tanto a la muerte del árbol, pero en verano podría tolerar hasta temperaturas de $45^{\circ} \mathrm{C}$ por lo que requiere de un clima templado con tendencia a cálido. Presenta dos floraciones al año: una pequeña llamada Sanjuanera y la principal que se inicia en setiembre y termina en diciembre; sin embargo, esta puede caer repentinamente si se producen lluvias fuertes o bajas temperaturas, a menos que las condiciones de tiempo favorezcan una segunda floración [6].
Sobre los meses en que se produce la floración del algarrobo, [7], identificaron los meses desde setiembre a marzo. En noviembre aparecen los botones florales y se hacen visibles. Las flores están maduras a finales de octubre y para especies recién instaladas la floración más abundante y estable se inicia al séptimo año. Después del fenómeno de "El Niño", en el año 1983, las floraciones han sufrido variaciones de un año a otro. Por ejemplo en los valles de Zaña y Chancay, la floración se inicia en diciembre, termina en febrero, mientras que en los valles de Motupe y Olmos es de octubre a diciembre [8]. En este aspecto el estudio fue desarrollado durante los años 2009 a 2012 tiempo que duro la observación.

\section{Materiales y Método}

El estudio fue realizado en las localidades de Mallares (Valle del rio Chira), Bernal (Valle del bajo Piura) y La Esperanza- Paita (valle del Chira), considerados lugares representativos en el desarrollo de la especie. Desde un punto de vista altitudinal la zona en estudio se encuentra distribuida entre los 0 metros sobre el nivel del mar a $45 \mathrm{msnm}$. Figura.1

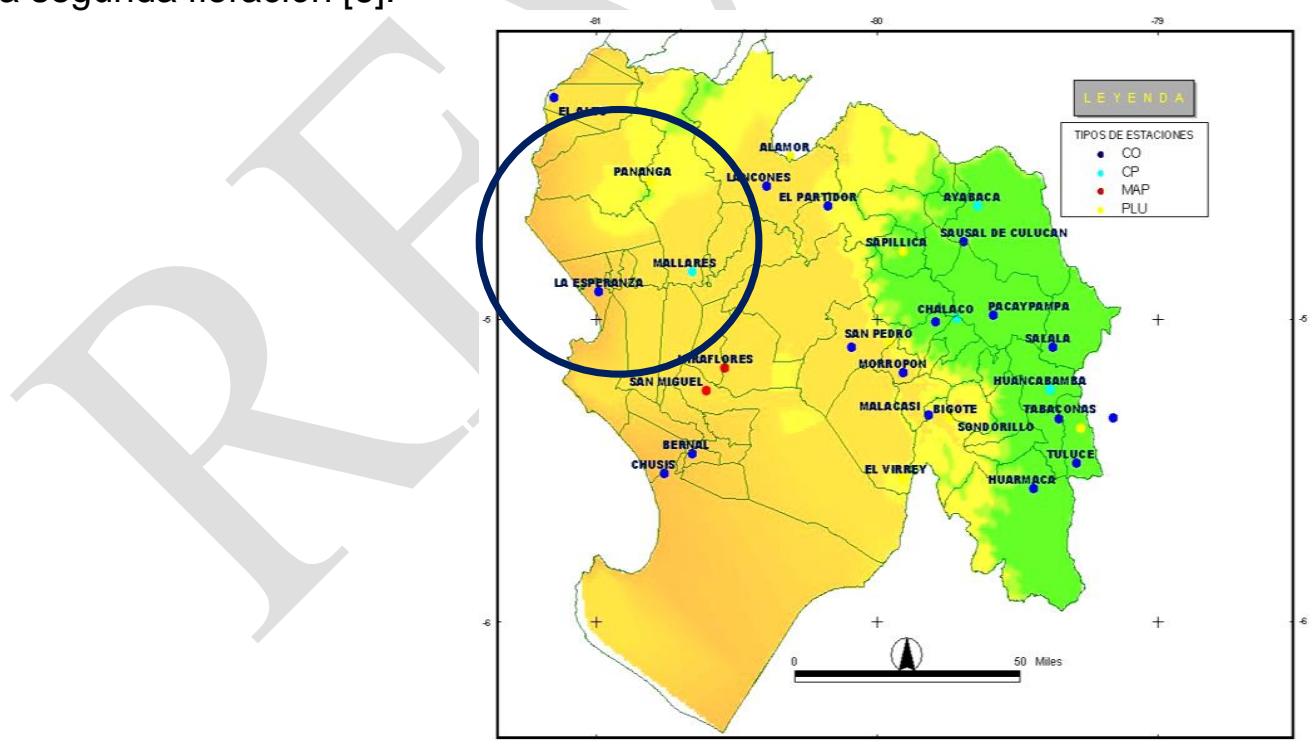

Figura 1: El área de estudio ámbito de la región Piura-Perú

De acuerdo al diagrama bioclimático de Holdridge (1982) [9]. el área de estudio presenta una temperatura media anual de $24^{\circ} \mathrm{C}$, y un valor promedio de precipitación total anual que varía entre 
81,0 y $260 \mathrm{~mm}$ incluido su estacionalidad marcada según el régimen de precipitaciones (con 9 meses secos y 3 meses de lluvias) presentando un constante déficit hídrico. De manera general, los sectores en estudio presentan un clima cálido característico de la zona de vida desierto Súper Árido Tropical. El contenido promedio anual de la humedad relativa del aire es de $73.76 \%$ [9].

Desde el punto de vista fenológico, es aplicada la metodología por copa que consiste en extraer la información sobre las lecturas de las floraciones de diez individuos previamente marcados y distribuidos aleatoriamente en cada una de las áreas destinadas a esta investigación procedente de la red de observación fenológica del SENAMHI. Cuando el 50 $\%$ de los ejemplares censados estaban en la fase buscada, se constituía la fecha media de iniciación de la fase. En este sentido es cuantificada la duración del estadio, en número de días. Los datos son procesados estadísticamente para un diseño totalmente aleatorio con 40 repeticiones determinándose diferencias significativas a través del test de Tukey $(\mathrm{P}<.0 .05)$.

Los datos meteorológicos, empleados, fueron extraídos de la base de datos procedente de la red meteorológica del Servicio Nacional de Meteorología e Hidrología del SENAMHI correspondiente a los años 1971-2012. Los valores sobre temperaturas diarias son analizados mediante técnicas de series de tiempo.
Grupo Morfológico. Los individuos analizados se encuentran enclavados dentro del ecosistema de bosque seco, representado por la especie Prosopis pallida. La clasificación morfológica de los árboles considera caracteres de flor en las etapas de inicio, pleno y fin. El periodo de floración se expresa en números arábigos que corresponden al calendario gregoriano. Estos datos corresponden a varias temporadas observadas de floración (años 20092012).

\section{Resultados y Discusión}

\subsection{Análisis de la Temperatura en los sectores observados}

En el ámbito del estudio, observamos una variabilidad en los resultados del registro de las temperaturas según el lugar de observación y en el tiempo. En el sector Mallares se observa un valor promedio de $26,17^{\circ} \mathrm{C} \quad(\mathrm{CV}=1,01 \%)$, con valores mínimo y máximo promedio de 19,3 y $31,4^{\circ} \mathrm{C}$, respectivamente. Estas fueron 1.86 y $1.53^{\circ} \mathrm{C}$ superiores, comparadas con las estimadas en el sector La Esperanza y Bernal. Se observa un importante incremento de las temperaturas durante los años 2008 al 2012 las diferencias fueron de $+1.61,+0,77$ y $+0,19^{\circ} \mathrm{C}$, respectivamente según las condiciones climáticas en los sectores Bernal, La Esperanza y Mallares. Ver Tabla 1.

Tabla 1: Condiciones climáticas en los sectores Bernal, La Esperanza, Mallares. (Valores promedios mensuales para el período 1971-2012).

\begin{tabular}{|c|c|c|c|c|c|c|c|c|c|c|c|c|c|}
\hline Sectores & Meses & Ene & Feb & Mar & Abr & May & Jun & Jul & Ago & Sep & Oct & Nov & Dic \\
\hline \multirow{3}{*}{ Bernal } & \multirow{2}{*}{$\begin{array}{l}\text { Temperatura } \\
\text { promedio } \\
{ }^{\circ} \mathrm{C}\end{array}$} & 32.8 & 33.6 & 33.3 & 31.9 & 29.4 & 27.4 & 26.8 & 26.9 & 27.8 & 27.9 & 29.4 & 30.9 \\
\hline & & 21.8 & 22.7 & 22.2 & 20.7 & 18.8 & 18 & 17.4 & 17 & 16.7 & 16.7 & 17.4 & 9 \\
\hline & $\begin{array}{l}\text { Precipitación } \\
(\mathrm{mm})\end{array}$ & 22.1 & 24.4 & 26.5 & 3.9 & 0.53 & 0.2 & 0.15 & 0.13 & 0.34 & 0.25 & 0.46 & 2.21 \\
\hline \multirow{3}{*}{$\begin{array}{l}\text { La } \\
\text { Esperanza }\end{array}$} & \multirow{2}{*}{$\begin{array}{l}\text { Temperatura } \\
\text { promedio } \stackrel{\text { C }}{ }\end{array}$} & 30.1 & 31.3 & 31.2 & 30.6 & 29 & 26.9 & 25.9 & 25.3 & 25.7 & 25.9 & 26.8 & 28.5 \\
\hline & & 21.9 & 23.3 & 23 & 21.8 & 20.1 & 18.8 & 17.9 & 17.4 & 17.5 & 18 & 18.6 & 20.2 \\
\hline & $\begin{array}{l}\text { Precipitación } \\
(\mathrm{mm})\end{array}$ & 18.2 & 23.3 & 32.2 & 25.4 & 19 & 10.6 & 0.06 & 0.06 & 0.1 & 0.48 & 0.45 & 4.34 \\
\hline \multirow{3}{*}{ Mallares } & \multirow{2}{*}{$\begin{array}{l}\text { Temperatura } \\
\text { promedio } \\
{ }^{\circ} \mathrm{C}\end{array}$} & 33.7 & 34.1 & 34 & 33.1 & 31.2 & 29.3 & 28.7 & 29 & 29.9 & 30.5 & 31.1 & 32.5 \\
\hline & & 21.6 & 22.5 & 22.1 & 20.8 & 19.4 & 18.2 & 17.1 & 16.9 & 17.1 & 17.6 & 18.3 & 19.6 \\
\hline & $\begin{array}{l}\text { Precipitación } \\
(\mathrm{mm})\end{array}$ & 37.3 & 59 & 84.4 & 46.6 & 19.1 & 0.41 & 0.24 & 0.19 & 0.18 & 1.28 & 1.15 & 9.9 \\
\hline
\end{tabular}


Revista ECIPerú

Según la Tabla 1, la temperatura mensual promedio más alta $\left(29,15^{\circ} \mathrm{C}\right)$ ocurrió entre enero a abril, en Mallares mientras que la más baja $\left(16.75^{\circ} \mathrm{C}\right)$, entre junio a octubre registrada en Bernal. Sobre el comportamiento de las temperaturas máximas, se observaron que siguieron el patrón cenital (equinoxios) que osciló entre 27 y $36^{\circ} \mathrm{C}$ como las registradas en la Esperanza-Paita y Mallares. En cuanto a la temperatura mínima promedio anual se mantuvo entre 18 a $20^{\circ} \mathrm{C}$. Asimismo, la figura 2, analizada muestra en el ámbito estudiado una amplitud anual (tempmax-tempmin) promedio de $9,06^{\circ} \mathrm{C}$ que osciló entre 9,3 y $12,10^{\circ} \mathrm{C}$.

\section{, número 1}

\subsection{Series de Tiempo}

Julio 2017

La Figura 2, muestra las series de tiempo de los valores diarios de la temperatura para las estaciones Mallares, Bernal y La Esperanza durante los años 2008 a 2012. Los valores observados presentan un comportamiento estacional, a excepción de un ligero incremento de las temperaturas mínimas entre mayo a agosto del 2012.

En general, durante el periodo observado, las temperaturas mínimas tendieron a ser más altas en los sectores Mallares seguida de La Esperanza y finalmente en el distrito de Bernal.

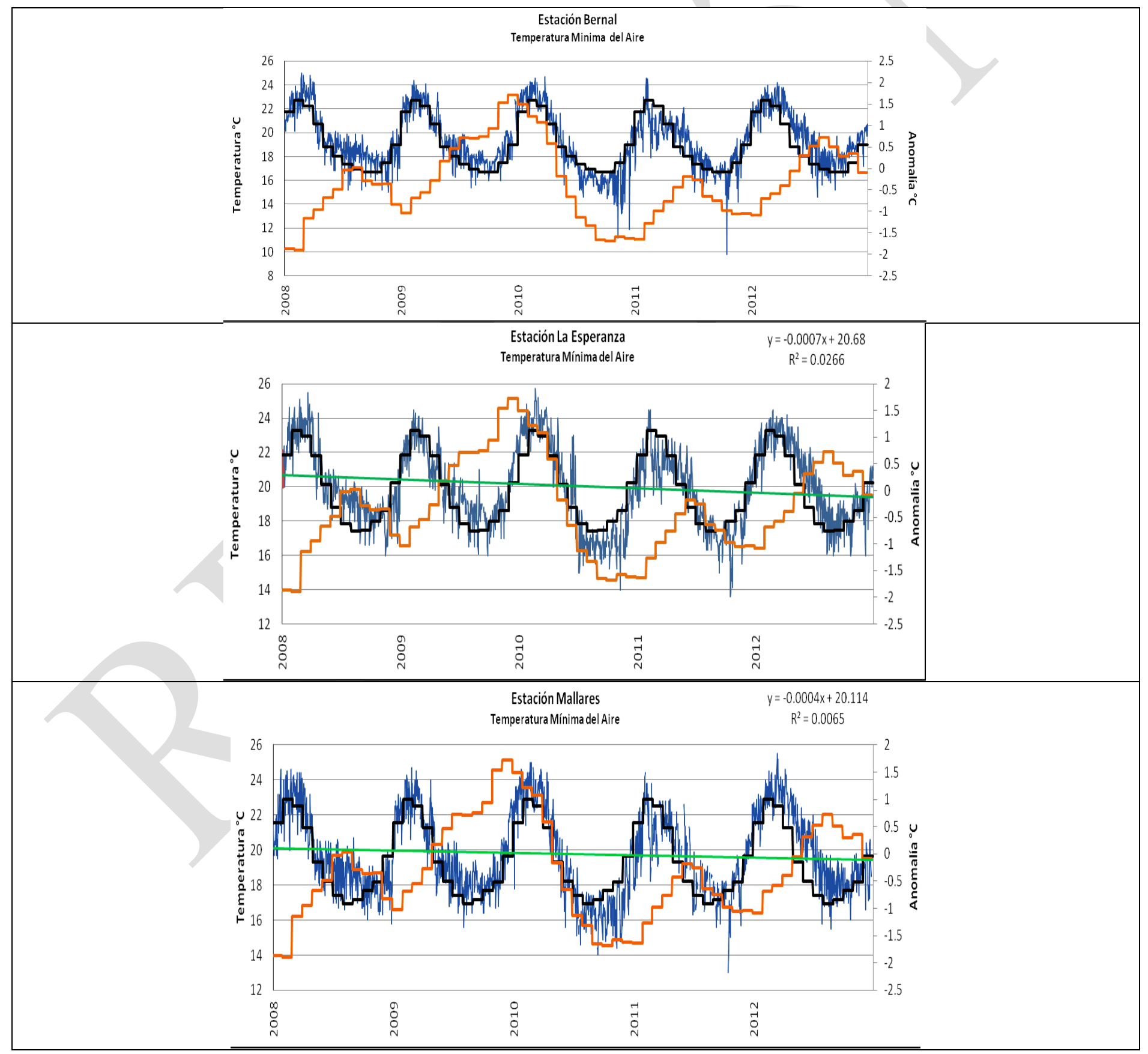


Revista ECIPerú

Volumen 14, número 1

Julio 2017

Figura 2: Series de tiempo diario de Temperatura mínima y Anomalías mensuales de la Temperatura superficial del mar TSM en el área 3.4, durante los años 2008-2012 en Mallares, Bernal y La Esperanza.

En cuanto a su variación mensual en las estaciones, Mallares, Bernal y La Esperanza, registraron 6 meses con una tendencia lineal hacia temperaturas mínimas más bajas, siendo marginalmente significativa solo para octubre y noviembre $(\mathrm{P}<0.05$. En cuanto a su distribución los datos observados, reportaron patrones muy similares (Fig 2.), sin embargo, se encontraron diferencias entre los promedios de las temperaturas mínimas de 8 meses $(P<0,05, n=11)$, excepto para enero, febrero, marzo donde prevalecieron las máximas temperaturas. Figura 3.

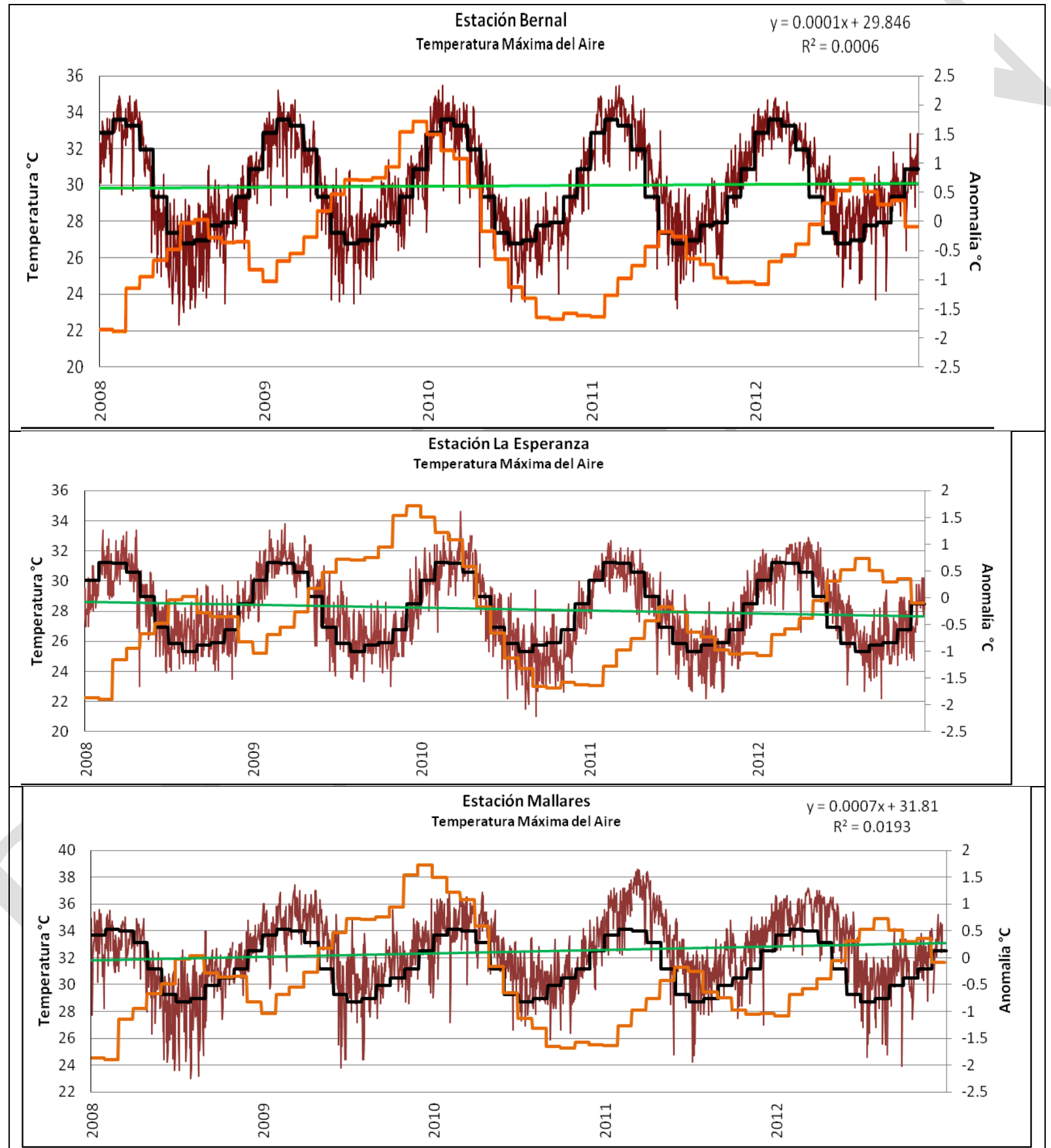

Figura 3: Series de tiempo diario de Temperatura máxima y Anomalías mensuales de la Temperatura superficial del mar TSM en el área 3.4, durante los años 2008-2012 en Mallares, Bernal y La Esperanza. 
De acuerdo a la figura 3, una observación interesante, es la coincidencia en las altas temperaturas registradas (entre los 30 y $35^{\circ} \mathrm{C}$ ), en otoño del 2012 para los tres sitios de estudio. Una posible explicación de este fenómeno generalizado para la región se corresponde con la influencia de las variaciones de la Temperatura Superficial del Mar TSM en el área niño 1+2, asociada al fortalecimiento del Anticiclón del Pacifico Sur APS durante algunos periodos. Se observa al mismo tiempo la tendencia a la normalización en el área niño 3.4. Dicha condición influenció sobre la aparición de flores en el algarrobo ocurridos en el sector Mallares correspondiente a la zona de mayor temperatura registrada en el ámbito de estudio y que provocaron alteraciones en el ciclo de floración en el algarrobo. Para el caso de la estación Mallares, se observó una frecuencia de por lo menos cada 6 meses (2008 a 2012), en que la temperatura muestra variaciones críticas en la época cálida.

\subsection{Análisis sobre el Periodo de floración}

La Figura 4 presenta el resultado del análisis sobre el periodo promedio (meses/año) de la floración del algarrobo en los sectores estudiados. Existe una variabilidad en la duración e inicio de la floración de un año a otro. El año 2009 se presentó con una floración reducida, desde un mes de floración en Bernal y La Esperanza a 4 meses de floración en el valle del Chira sector Mallares en relación a los demás valles analizados.

Entre los años 2010, 2011 y 2012 se reporta de manera progresiva un incremento y una mayor frecuencia en el tiempo de floración, concentrados entre septiembre a enero. De esta manera, los individuos que fueron los primeros en florecer en un año, a la temporada siguiente también lo hicieron, es el caso de aquellos ubicados en La Esperanza-Paita donde se registró floración hasta el mes de enero durante los años 2009 a 2012. El mismo comportamiento fue registrado en aquellos sectores que florecían tardíamente como lo observado en Bernal.

En cuanto a la cantidad de flores, el año 2012 en los sectores observados, existió un tiempo más prolongado de floración traducido en un menor número de flores por individuo. Figura 4.

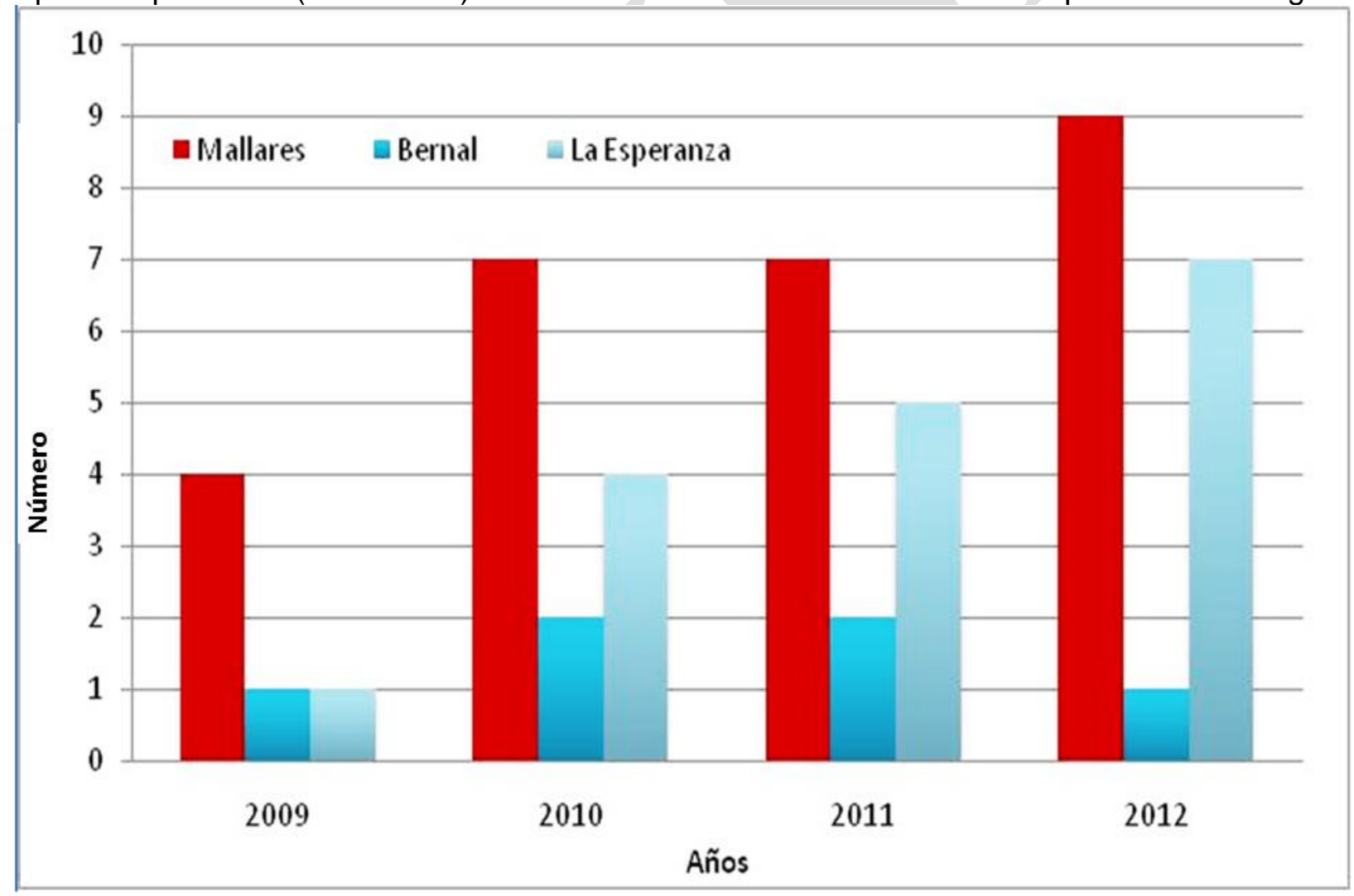

Figura 4: Distribución del tiempo (meses) de floración 
En este mismo análisis, el estadio de floración presenta un amplio rango de posibilidades (desde un mes en Bernal el año 2009 a 9 meses en Mallares el año 2012), lo que significa que un solo individuo puede generar flores durante un prolongado tiempo, condición observada con mayor frecuencia en Mallares, La Esperanza y Bernal respectivamente.

\subsection{Respuesta Estacional de la Floración}

La variación estacional es considerada dentro de un patrón periódico en una serie de tiempo. La figura 5, muestra que la respuesta floral es relativamente

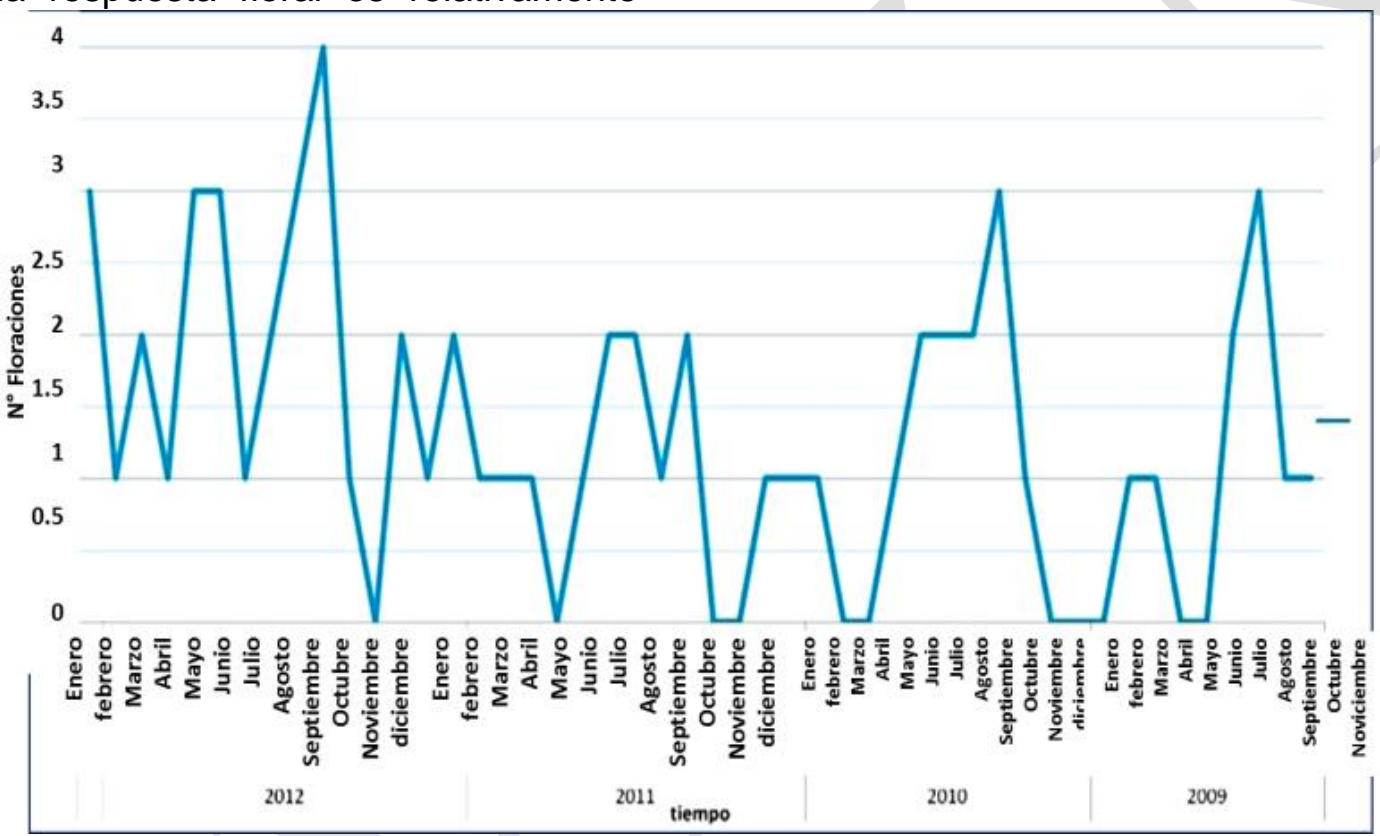

Figura 5: Comportamiento estacional de la floración del algarrobo entre los años 2009-2012 en Mallares.

De la figura 5, el año 2009 en Mallares, fueron reportadas floraciones en tres frecuencias entre enero, mayo-junio y septiembre. En la zona de La Esperanza-Paita, no se observa floración en abril mientras que en la zona de Bernal, la floración se produjo durante los meses de septiembre a enero. De manera general, dichos resultados presentan coincidencia con los estudios realizados por autores como Digilio y Legname (1966) [10]. quienes indican que "la mayoría de las leñosas florecen a fines de invierno y comienzos de primavera. Estos mismos autores manifiestan que cuando los inviernos son largos y más severos que lo acostumbrado, la floración se retrasa. Por lo que, en ninguno de los casos se observa en Mallares, presencia de floración en el mes de julio. De esta manera, el uso de la oferta ambiental de flores estuvo condicionado en casi todos los casos cuando la temperatura superaba los $16^{\circ} \mathrm{C}$. Existen datos empíricos donde indican que la especie se considera como foto periódica. estacional, observándose una mayor abundancia en la estación de primavera que exhibe una distribución amplia en el terreno. Su estacionalidad de la floración del algarrobo, oscila entre mediados de agosto a fines de enero, pero en algunos años se observa que se apartan significativamente de este período. Se observa, una respuesta variada durante el inicio de la fase de floración dependiendo del lugar y el año. Aunque la mayoría de autores reportan que Prosopis pallida florece dos veces al año, hemos encontrado una respuesta distinta en los periodos de observación. Figura 5. 


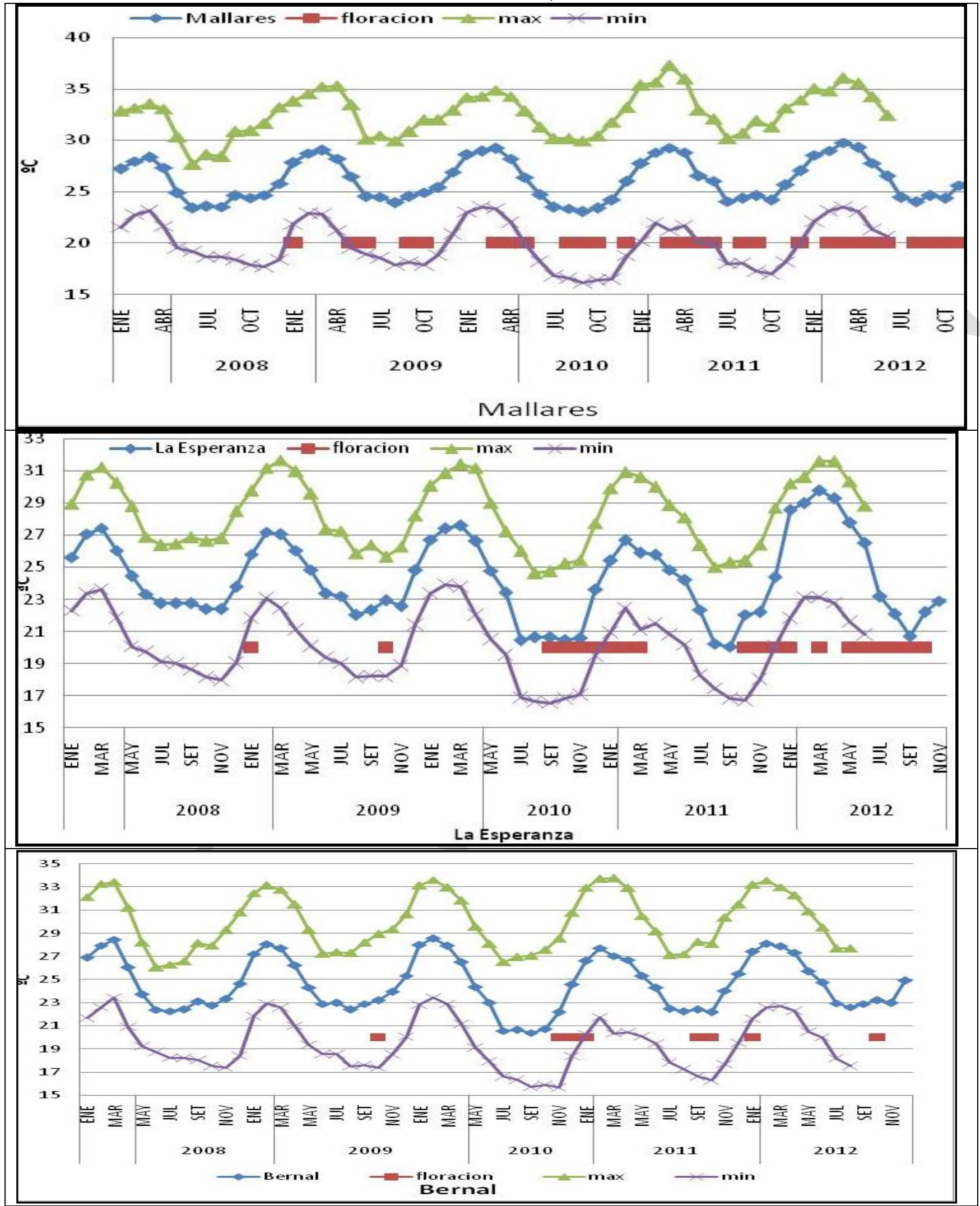

Figura 6: Distribución de las temperaturas y tiempos de floración del algarrobo en Mallares, Bernal y La Esperanza años 2009-2012. 


\section{Discusión}

La metodología por copa permitió el seguimiento de la fase de floración, siendo necesario realizar un entrenamiento previo del observador para la toma de datos, ya que se asignan porcentajes subjetivos a cada fenofase. En cuanto al impacto de la temperatura en la producción de flores observamos la acción directa o indirecta de la temperatura, siendo necesario incorporar una variable complementaria para fortalecer el resultado como es la precipitación. Al ser el género Prosopis, de gran importancia por su uso como melífero, el momento de máxima abundancia en la floración es de corta duración con un rango conformado por sectores más tempranos y más tardíos.

\section{Conclusiones y Recomendaciones}

El seguimiento fenológico que permitió registrar la floración del algarrobo depende del lugar y de la temperatura, lo cual se traduce en las relaciones de temperatura y floración. Se observa en este estudio que existe una mayor frecuencia de floración en Mallares y La Esperanza donde las temperaturas se presentan más elevadas que en Bernal por lo que la ubicación geográfica influye sobre este proceso.

De otro lado, el flujo de anomalías en la temperatura como consecuencia de la influencia del fenómeno "La Niña", asociada al descenso de las temperaturas, quedó reflejada en la respuesta estacional de la floración del algarrobo tal y como sucedió durante los años 2009-2011, donde la especie ofreció en distintas condiciones ambientales, diferencias significativas en el periodo de floración.

Se observa un amplio rango de floración en el tiempo donde el momento de máxima abundancia en la floración se presenta de corta duración, lo cual constituye una de las limitantes de las especies de Prosopis para su aprovechamiento como especies melíferas especialmente en sectores de menor abundancia como es Mallares. Sin embargo, esta deficiencia se compensaría con la variabilidad de la especie según su ubicación geográfica y su desplazamiento fenológico. Se destaca en este análisis que el sector de Mallares presenta mayor cantidad de días con floración, pero con menor carga floral.

En cuanto a la metodología por copa aplicada esta resultó ser un método rápido aunque para profundizar el estudio se recomienda realizar una observación detallada a nivel de ramas.

Los resultados obtenidos en este trabajo tienen significativas coincidencias, con los estudios encontrados por [13], [14], [15], [16], [17], [18],

Debido a los mecanismos de adaptación de la respuesta floral ante la variabilidad en el clima, esta especie en particular ha registrado mayor floración ante el incremento en la temperatura aspecto importante en la producción y como potencial del ámbito de desarrollo de la biodiversidad que se desarrolla en el bosque.

\section{Referencias}

[1] R.A. Mittermeier; P. Robles Gil; M. Hoffman; J. Pilgrim; T. Brooks, C. G. Mittermeier; J. Lamoreux and G. A. B. Da Fonseca. "Hotspots Revisited: Earth's Biologically Richest and most Threatened Terrestrial Ecoregions". Conservation International, Washington. (2005). 392

[2] $F$ Roig .Observaciones fenológicas del Algarrobo Negro (Prosopis Flexuosa D.C.) y del algarrobo blanco Proposipis Chilensis (MOL.) STUNTZ). En el Chaco Árido, ArgentinaE. (1993).

[3] A. Silva \& Higaona. Fenología de las principales especies forrajeras de las Pampas de Olmos. En Centro Internacional de Investigación para el Desarrollo 1991. Investigación sobre Forrajeras Xeróftas y Capricultura (1984 - 1989). Lima, Peru

[4] M. I. Borchert, F. W. Davis. J. Michaelsen \& L. Oyler, Interaction of factors affecting seedling recruitment of blue oak (Quercus douglasii) in California. Ecology (1989).70: 389-404.

[5] S. Bridgewater, R.T. Pennington, C.A. Reynel, A. Daza y T. Pennington. A preliminary floristic and phytogeographic analysis of the woody flora of seasonally dry forests in northern Peru. Candollea (2003). 58: $129-148$

[6] P. L Hurtado. Estudio de prefactibilidad para la instalación de un apiario en la irrigación San Lorenzo. Piura. Tesis UNALM. Lima, (1981). 344 pp.

[7] J.R. Martos, M. Scarpati, C.Rojas \& G.E. Delgado Fenología de algunas especies que son alimento para la pava aliblanca 
Penelope albipennis. Revista Peruana de Biología (2009)15: 51-58.

[8] FAO. State of the World's Forests, 1997.

[9] SENAMHI. Boletín Meteorológico, (2012). Vol 5, 12. 21. Pág.

[10] L.A. Fournier. Algunas observaciones sobre la dinámica de la floración en el bosque tropical húmedo de Villa Colón. Rev. Bío!., Trop.,1980. 14(1) : 75-85,

[11] A. Carvajal, Cafeto: cultivo y fertilización. Instituto Internacional de Potasa. Berna, Suiza.1984. p. 254.

[12] A. Segura. Estimación del efecto del fenómeno de "El Niño" sobre el crecimiento y la producción de café de San Marcos de Tarrazú, 1997-1998: Un enfoque fisiológico a un problema producido por un 68éficit hídrico. Esc. De Fitotecnia, Universidad de Costa Rica. (mimeografiado, s.p.). 1997.

[13] J.H. Morello, y C. Saravia ToledO. "EI bosque chaqueño: I. Paisaje primitivo, paisaje natural y paisaje cultural en el Oriente de Salta". Rev. Agron. del Noroeste Argentino 1959. 3 (1-2): 5-81 y 209-258; Tucumán, Argentina.
[15] N.R. Ledesma, y Medina J.C. "Fenología de la comunidad forestal Chaco santiagueña". Actas del lo Congreso Forestal Argentino; Bs. As., Argentina (1969)." Vol. I: 801-806.

[16] P.R. Legname, "Árboles indígenas del Noroeste Argentino". Opera Lilloana 34: 226 p.; Tucumán, Argentina.(1982).

[17] Dostert, N.; Roque, J.; Brokamp, G.; Cano, A.; La Torre, M. \& Weigend M. 2009. Factsheet: Datos botánicos de Algarrobo Prosopis pallida. Proyecto Peru Biodiverso PBD. Lima, Perú. 10 pp.

[18] FM. Galera. Las especies del género Prosopis (algarrobos) de América Latina con especial énfasis en aquellas de interés económico. Departamento de Agricultura y Protección del Consumidor, Organización de las Naciones Unidas para la Alimentación y la Agricultura (FAO). Versión en línea: http://www.fao.org/docrep/006/ ad314s/ad314s00.htm [03.08.2011. (2000).

[14] A. P. L. Digilio \& P. R. Legname. Los árboles indígenas de la provincia de Tucumán. Opera Lilloana 15: 54.(1966). 